\title{
REPERCUSSÕES DA IMERSÃO SOBRE A PRESSÃO ARTERIAL EM GESTANTES
}

\author{
GRAD. LEOMARA MENDES DE OLIVEIRA
}

Bacharel em Fisioterapia pela Universidade Federal da Paraíba (Paraíba - Brasil) e-mail: leomaraoliveira@hotmail.com

\section{MS. MALLISON DA SILVA VASCONCELOS}

Doutorando em Saúde Materno-Infantil pelo Instituto de Medicina Integral Prof. Fernando Figueira (IMIP), Mestre em Fisiologia pela UFPE e Professor Assistente do

Departamento de Fisioterapia da Universidade Federal da Paraíba (Paraíba - Brasil) e-mail:continencia@hotmail.com

\section{MS. SANDRA MARIA CORDEIRO ROCHA DE CARVALHO}

Mestre em Educação pela UFPB e Professora Assistente do Departamento de Fisioterapia da Universidade Federal da Paraíba (Paraíba - Brasil) e-mail: sandracordeiror@yahoo.com.br

\section{MS. MARIA DO SOCORRO NUNES GADELHA}

Doutoranda em Administração Hospitalar e Sanitária UEx (Espanha), Mestre em Serviço Social pela UFPB e Professora Assistente do Departamento de Fisioterapia da Universidade Federal da Paraíba (Paraíba - Brasil) e-mail: gadelhasocorro@hotmail.com

\section{RESUMO}

Revisamos na literatura as modificações da PA em gestantes durante a imersão para compreender os fatores que influenciam esse comportamento. Observa-se que a PA de repouso é reduzida com a imersão em meio aquático, sendo esta redução proporcional ao nível de imersão. Fatores como temperatura da água, posição corporal, profundidade e tempo de imersão influenciam nas respostas fisiológicas do organismo a essa condição. Contudo, os estudos apresentam diferentes parâmetros para tais variáveis, o que dificulta o estabelecimento de uma adequada indicação desta modalidade como recurso terapêutico.

PALAVRAS-CHAVE: Gravidez; hipertensão induzida pela gravidez; fisioterapia. 
As síndromes hipertensivas durante a gravidez são a segunda causa principal da mortalidade materna, complicando de $5 \%$ a $8 \%$ de todas as gestações (SORENSEN et al., 2003; PASCOAL, 2002; MINISTÉRIO DA SAÚDE, 2006). A morbimortalidade ocorre em torno de 12 a 22\% das gestações, sendo responsável por 35\% de mortes maternas no Brasil (PERAÇOLI; PARPINELLI, 2005).

A pré-eclâmpsia/ eclampsia continua sendo a primeira causa de mortalidade materna, determinando um maior número de óbitos perinatais, além do aumento significativo do número de neonatos com sequelas, quando estes sobrevivem aos danos da hipóxia cerebral (MINISTÉRIO DA SAÚDE, 2006; HERMIDA; AYALA; IGLESIAS, 200I). Tal condição é específica da gravidez, envolve a falência de múltiplos órgãos e está associada à hipertensão e proteinúria, as quais aparecem a partir da 20ª semana de gestação (CAVALLI et al., 2009).

A invasão do citotrofoblasto nas artérias espiraladas durante o início do segundo trimestre é fator determinante para a criação de um sistema de baixa resistência essencial para o desenvolvimento e crescimento fetal. Na pré-eclâmpsia, esta invasão é limitada ocorrendo entre 30 e 50\% das artérias, as quais apresentarão a metade do diâmetro de uma arteríola em uma gravidez normal, levando a uma diminuição da perfusão útero-placentária e consequente isquemia da placenta. A isquemia placentária induz a produção de substâncias vasoativas que promovem a disfunção do endotélio vascular em órgãos nobres resultando na hipertensão (GILBERT et al.,2008; CAVALLI et al., 2009).

tratamento com antihipertensivos na gestação com pré-eclâmpsia ainda gera controvérsias, pois ainda não são conhecidos os reais benefícios do mesmo, devendo ser realizado de forma cautelosa e somente nos casos moderados e graves (SOUZA; AMORIM; COSTA, 2008).

Nesse contexto, a prática regular de atividade física pode ser uma estratégia adotada para a prevenção do aumento da pressão arterial (PA) durante a gestação, já que mulheres fisicamente ativas apresentam redução do risco de desenvolver hipertensão gestacional em aproximadamente 35\% (SORENSEN et al., 2003). No entanto, algumas alterações do curso normal da gestação determinam contraindicações para o exercício físico em solo ou aquático pelo fato destes induzirem o risco de aborto e complicações clínicas (ACOG, 1994). Diante desse fato, a imersão na água surge como forma preventiva e intervencionista, sendo mais segura, prática e rápida para mobilizar líquidos extravasculares e reduzir a PA, sem a presença de efeitos colaterais sobre a mãe e o feto (KWEE et al., 2000; AYRES et al., 200।; KATZ et al., 1990). 
As vantagens da imersão na água estão associadas às suas características físicas. De acordo com Katz et al. (1990), durante a imersão a força hidrostática atua dentro de segundos e ativamente empurra o fluido extravascular para o espaço vascular, sendo esta força proporcional à profundidade da água. $\bigcirc$ aumento da pressão hidrostática provoca um aumento no retorno do sangue venoso para o sistema cardiovascular, fazendo aumentar a produção de peptídio natriurétrico atrial com consequente efeito sobre o débito urinário, reduzindo desta forma a PA e o edema (KATZ, 2003; AYRES et al., 200 I). Outra vantagem da imersão é que esta diminui a atração gravitacional, resultando em uma sensação de sentir-se mais leve. A diminuição do peso hidrostático também gera maior flutuação e o empuxo cria uma sensação de conforto físico, melhora a mobilidade e diminui a dor pós-exercício (FINKELSTEIN et al., 2004; SMITH; MICHEL, 2006).

A imersão em repouso ou atividade física na água está relacionada a inúmeros benefícios para as gestantes. Por isso, é importante uma melhor compreensão das alterações fisiológicas e do comportamento da PA na imersão em repouso, para que seja possível uma adequada indicação dessa modalidade como recurso terapêutico.

o objetivo do presente estudo foi revisar o comportamento da pressão arterial em gestantes durante a imersão e compreender os fatores que influenciam esse comportamento.

\section{METODOLOGIA}

Trata-se de uma revisão narrativa de artigos publicados sobre o referido tema, inseridos nos seguintes bancos de dados: MEDLINE, SCIELO, LILACS, PubMed, SCIRUS, Bireme Bioline Internacional e The Cochrane Library. Foram utilizadas para a captação dos artigos as seguintes palavras-chave: immersion, hypertension, pregnancy, physiotherapy, physical therapy.

Foram incluídos os estudos experimentais ou de revisão que abordassem as alterações hemodinâmicas e cardiovasculares em gestantes submetidas à imersão estática na água com ou sem a prática de exercícios físicos. Não houve preferência quanto ao idioma ou ano de publicação. Foram excluídos os artigos captados com as palavras-chave utilizadas na busca mas que não correspondiam ao escopo da pesquisa.

período de busca junto aos bancos de dados foi de janeiro a março de 20 I0. Inicialmente foram encontrados um total de 23 artigos, sendo I I selecionados ao término da pesquisa. Os estudos selecionados continham no título, pelo menos, uma das palavras-chave. Após identificação dos mesmos, foram agrupados por ordem cronológica (sem limitação de datas), temática discutida e enfoque, facilitando a análise. 
Colheram-se informações dos artigos relacionadas: ao tempo de imersão, à temperatura da água, ao comportamento da pressão arterial, às características da amostra, aos efeitos adversos, às indicações e contra-indicações, entre outras.

Quando de posse do material para análise e síntese, deu-se seguimento as seguintes etapas: leitura exploratória e reconhecimento dos artigos que interessavam à pesquisa; leitura seletiva, com escolha do material que, de fato, servia aos propósitos da pesquisa; leitura crítica e interpretativa em que foram procurados resultados, apresentados nos artigos, associados à necessidade do estudo e, por fim, confronto dos dados encontrados na análise.

\section{RESULTADOS}

Foram encontrados II estudos quantitativos e experimentais pertinentes ao tema pesquisado. Observou-se um grande volume de informações sobre os efeitos fisiológicos decorrentes da imersão, sobre a avaliação das alterações hemodinâmicas maternas durante esse procedimento, contudo apenas 4 estudos abordaram a imersão em gestantes de risco, sendo que apenas um destes, além da imersão, também abordou o exercício na água, nessa população. O período gestacional dos sujeitos avaliados correspondia ao $3^{\circ}$ trimestre de gestação em oito dos artigos selecionados.

A literatura consultada mostra de forma unânime a redução da pressão arterial média (PAM) na imersão, principalmente devido às propriedades físicas da água. Também encontraram-se informações sobre a influência da imersão no sistema renina-angiotensina, na vasopressina, no hormônio natriurético atrial (FAN), na diurese, no índice de líquido amniótico e na frequência cardíaca. Nenhum dos estudos apresentou danos à saúde materna e fetal.

\section{DISCUSSÃO}

\section{EFEITOS FISIOLÓGICOS DA IMERSÃO MATERNA}

A gestação leva a significativas alterações na estrutura, no metabolismo, na função endócrina e cardiovascular da mulher. Na gestação saudável, observa-se o aumento da frequência cardíaca (FC), do consumo de oxigênio, do débito cardíaco e do volume sistólico, permitindo um ajuste contínuo do volume sanguíneo ao leito vascular (FINKELSTEIN et al., 2004). Além disso, a PA diminui até a metade da gravidez e, por volta da $20^{a}$ semana, ocorre um aumento médio de $8 \%$ até o dia do parto (HERMIDA; AYALA; IGLESIAS, 200 I; COELHO; POLITO, 2009), sendo de 
fundamental importância a rotina na verificação da PA, já que a hipertensão induzida pela gravidez está relacionada à morbidade e mortalidade materno-fetal (KOKOT; ULMAN; CEKANSKI, 1983).

Durante a gravidez normal, ocorrem mudanças hemodinâmicas para fornecer um fluxo sanguíneo uteroplacentário adequado. Durante o $1^{\circ}$ trimestre, ocorre o aumento do débito cardíaco, o volume de plasma aumenta em 40\%, enquanto a resistência vascular sistêmica diminui. Na gestação complicada por pré-eclâmpsia ou retardo no crescimento uterino, essas mudanças fisiológicas são menores ou não são mantidas. Nessas condições, a utilização de imersão objetivando corrigir essas más adaptações, pode ser benéfica para mãe e para o feto (KWEE et al., 2000).

As forças físicas da água, agindo sobre um corpo imerso, levam a alterações fisiológicas extensas, influenciando quase todos os sistemas do organismo. Segundo a Lei de Pascal, a pressão hidrostática é definida como a força exercida por unidade de área, ou seja, é a pressão do líquido sobre o corpo imerso. Enquanto o empuxo é uma força de flutuação que atua no sentido oposto à força da gravidade, correspondendo ao peso do líquido deslocado. Dessa forma, o corpo na água sofre a ação de duas forças que estão em oposição: a força de gravidade e a força de flutuação (CAROMANO; NOWOTNY, 2002).

Se a gestante estiver em pé na água ou submersa, seu corpo sofre uma pressão maior nos membros inferiores, com alterações nos volumes dos líquidos intra e extravasculares, auxiliando o retorno venoso e com consequente débito cardíaco maior (AYRES et al., 200 I). Também ocorre o redirecionamento sanguíneo que corrobora com o aumento da diurese por meio da estimulação dos receptores que promovem ajustes hormonais e neurais, os quais conduzem a uma queda na PA sistólica e diastólica (HARTMANN., 200 I). Em conjunto, todas estas propriedades resultam em uma correção postural e do edema da grávida, na redistribuição dos fluidos corporais e em um maior aporte de nutrientes e fluxo ao feto (AYRES et al., $200 \mathrm{l}$ ).

A imersão também aumenta o volume de sangue central em até $700 \mathrm{~mL}$, que por sua vez, promove um aumento do peptídio natriurético atrial (PNA), responsável por suprimir o sistema renina-angiotensina-aldoesterona (RAA) e o sistema nervoso simpático (SNS), levando à diminuição da pressão sanguínea (WARD et al., 2005).

Grafa (1986) ressalta que as mulheres devem ser encorajadas a imergir na água da piscina de, pelo menos, 30 a $32^{\circ} \mathrm{C}$, para minimizar os riscos de desenvolver pré-eclâmpsia, pois a água produz uma sensação de ausência de peso, diminui o peso corporal total em dez por cento (10\%) e diminui os esforços às articulações e ligamentos; sendo assim um ambiente ideal para o exercício. 
Está bem consolidado na literatura que a PA de repouso sofre influência com a imersão em um meio líquido. Entretanto, os estudos que a analisaram apresentam diferentes parâmetros no que se refere à temperatura da água, ao tempo de imersão e ao nível de imersão (KWEE et al., 2000; KATZ, 2003; FINKELSTEIN et al., 2006; KOKOT; ULMAN; CEKANSKI, 1983; ASAl et al., 1994; HARTMANN e HUCH, 2005; ELVAN-TASPINAR et al., 2006; AYRES et al., 200 I).

A variação da PA de repouso no meio líquido depende da temperatura da água, da posição corporal, da profundidade de imersão e da PA inicial (ALBERTON; KRUEL, 2009; WARD. , 2005). Assim, pequenas mudanças na temperatura da água têm efeitos significativos sobre a perda de calor ou retenção de calor no paciente imerso, porque a água tem uma condutividade térmica 25 vezes maior que o ar (KATZ; MCMURRAY; CEFALO, 1991). Além de que temperaturas acima de $38,9^{\circ} \mathrm{C}$ são perigosas na imersão durante a gravidez, principalmente no $1^{\circ}$ trimestre, pois podem provocar malformações embrionárias ou fetais (ROGERS; DAVIS, 1995).

Kokot, Ulman e Cekanski ( 1983 ) relataram uma queda rápida e acentuada da pressão arterial sistólica ( | 46,33 \pm 3,05 mmHg no início para | 27,55 \pm 2,29 $\mathrm{mmHg}$ aos 60 minutos) e diastólica (93,77 \pm I,38 no início para 80,44 \pm 1,86 $\mathrm{mmHg}$ aos 60 minutos), significativamente maior no grupo de gestantes com pré-eclâmpsia $(n=45)$ do que no grupo de gestantes saudáveis $(n=39)$, além de significativa diminuição na atividade da renina plasmática, aldosterona e vasopressina em ambos os grupos na imersão em água a $35-36{ }^{\circ} \mathrm{C}$ até a profundidade do pescoço. No entanto, não foi encontrada correlação significativa entre as alterações da pressão arterial e os padrões de reação endócrina induzida pela imersão na água, indicando que fatores desconhecidos também estão envolvidos na patogênese da hipertensão induzida pela gravidez.

Nesse sentido, Ivanov e Markova (1990) avaliaram o efeito da imersão em hipertensos durante crise hipertensiva, demonstrando que após 90 minutos de imersão houve queda da PA, devido a benéficas alterações hemodinâmicas e hormonais, concluindo que este método terapêutico produz um efeito calmante e diurético, provando ser altamente eficaz no tratamento de crises hipertensivas de diferentes tipos, além de não produzir efeitos secundários, podendo ser usado em um hospital com boa relação custo-benefício.

Não obstante, Katz et al. (1990) comparando três tratamentos: repouso, imersão ao nível da cintura pélvica, imersão ao nível da cintura escapular para edema em I I gestantes saudáveis, com média de 30 anos, verificaram que em todos 
os tratamentos ocorreu declínio da PA, sendo este mais significativo na imersão em água ao nível da cintura escapular $(70 \pm 9 \mathrm{mmHg})$, aos 50 minutos de imersão, comparado com $81 \pm 10 \mathrm{mmHg}$ no repouso e $79 \pm 11 \mathrm{mmHg}$ na imersão até a cintura pélvica, à temperatura de $32 \pm 0,5^{\circ} \mathrm{C}$, o que mostra a influência da profundidade de imersão sobre a queda da PA. Contudo, cabe salientar que a temperatura da água nesse estudo foi abaixo da temperatura considerada como termoneutra para repouso $\left(34-35{ }^{\circ} \mathrm{C}\right)$, que foi utilizada em alguns estudos (KATZ et al. 1992; AIRES. 200 I; KWEE et al. 2000; ELVAN-TASPINAR et al. 2006).

Asai. ( 1994) examinaram 20 gestantes saudáveis sob três condições: repouso em terra, imersão estática e exercício na água. Os resultados indicaram que a vasopressina diminuiu durante a imersão em água e aumentou após o exercício; a atividade da renina plasmática diminuiu nas duas últimas condições e a concentração do peptídeo natriurético atrial (PNA) aumentou significativamente após o exercício na água, levando os autores a concluirem que a diminuição da PA e aumento do volume de urina durante o exercício foram causados pela diminuição da atividade da renina plasmática e pelo aumento da concentração do PNA, o que resultou na expansão do volume de sangue durante o exercício na água. No entanto, os autores sugeriram uma melhor investigação da resposta endócrina após o exercício na água, devido à divergência com os resultados encontrados em outros estudos.

Esse achado corrobora com Doniec-Ulman et al. (1987) que mostraram uma queda rápida na monitorização ambulatorial da pressão arterial (MAPA) com a imersão na profundidade da cintura escapular em todos os grupos analisados, sendo a diminuição da pressão arterial média (PAM) máxima obtida após I hora de imersão, com simultânea diminuição da atividade da renina, aldosterona e vasopressina plasmática e um aumento do PNA, no entanto, as mudanças induzidas pela imersão sobre a PA não foram relacionadas de forma significativa com as alterações dos fatores hormonais acima mencionados.

Kwee et al. (2000) analisaram as respostas hemodinâmicas de 19 gestantes saudáveis em imersão na água na profundidade de I, 10 m a I,70 m, em temperatura termoneutra, durante 35 minutos. Comparado com o repouso absoluto e os valores pré-imersão, a imersão resultou em uma diminuição significativa da PA, que retornou aos valores basais dentro de I,5 horas, mostrando um efeito claro, mas de curta duração. Na idade gestacional (IG) de 26-29 semanas, a PAM foi de $83,6 \mathrm{mmHg}$, sem imersão e 76,2 mmHg durante a imersão. Mais adiante, na IG de 34-37 semanas, estas valores foram de $89,1 \mathrm{mmHg}$ e $79,8 \mathrm{mmHg}$, respectivamente $(p<0,00 \mathrm{I})$.

Elvan-Taspinar et al. (2006) avaliaram o efeito da imersão em água durante 3 horas sobre a hemodinâmica central e periférica, em temperatura termoneutra, de 7 
gestantes com pré-eclâmpsia, de 7 gestantes saudáveis e de 7 mulheres não-grávidas. A imersão em água fez aumentar o débito cardíaco e reduzir a frequência cardíaca, a PA diastólica e a resistência periférica total, mas os resultados foram limitados e transitórios. Contrariando os outros estudos, a PA sistólica manteve-se inalterada em todos os grupos. Mesmo com o declínio, a resistência periférica total manteve-se maior em mulheres com pré-eclâmpsia do que seus pares normotensos. Além de que a imersão em água não teve efeito na vasodilatação do endotélio-dependente no grupo de pré-eclâmpsia, e a maioria das alterações hemodinâmicas observadas revertiam-se ao valor basal até 2 horas após a conclusão do procedimento. Por isso, os investigadores concluíram que é pouco provável que a imersão total em água seja útil como uma modalidade terapêutica na pré-eclâmpsia, embora seja teoricamente aceita, o seu potencial terapêutico é limitado e de curta duração. Vale salientar que a amostra apresentou um número de participantes reduzido, o que pode ter influenciado nos resultados.

Em estudo semelhante de Hartmann et al. (200 I), a imersão, à temperatura média de $29^{\circ} \mathrm{C}$, afetou minimamente a PA e todos os valores desta foram consideravelmente menores após o exercício na água do que em repouso em terra e em água. Todavia, Hartmann e Huch (2005) observaram um aumento significativo

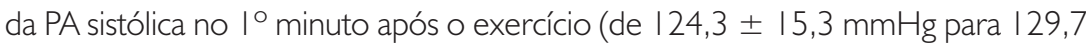
\pm । 4,8 $\mathrm{mmHg}, p=0,049$ ), enquanto a PA diastólica permaneceu praticamente inalterada, em uma única sessão de imersão à temperatura média de $30,9^{\circ} \mathrm{C}$.

\section{INFLUÊNCIA DA TEMPERATURA, PROFUNDIDADE E TEMPO DE IMERSÃO}

É importante notar que os parâmetros de temperatura da água, umidade e profundidade de imersão devem ser os mesmos para que se obtenham os mesmos resultados, contudo os estudos mostram padrões semelhantes nas alterações da pressão arterial na imersão de gestantes em piscinas cujas temperaturas variaram entre 30 ${ }^{\circ} \mathrm{C}$ a $36{ }^{\circ} \mathrm{C}$ (KOKOT; ULMAN; CEKANSKI, 1983; DONIEC-ULMAN et al., 1987; KATZ et al., 1990; HARTMANN e HUCH, 2005; WARD et al., 2005).

Com relação à profundidade de imersão, o nível da cintura escapular foi o mais utilizado e no qual se obteve os melhores resultados na diminuição da PA, ratificando a ação da pressão hidrostática sobre o sistema cardiovascular (ELVAN-TASPINAR et al. 2006; HARTMANN e HUCH, 2005; HARTMANN et al., 200 I; KATZ et al. 1990; AYRES et al., 200 I; FINKELSTEIN et al., 2006).

O tempo de imersão variou de 35 minutos até três horas e na maioria dos estudos $(n=10)$ foi realizado em uma única sessão, o que sugere que para a obtenção de mudanças duradouras nos eventos hemodinâmicos, as gestantes 
devam ser submetidas à imersão por um período mais longo ou mais de uma vez ao dia, ressaltando a mensuração da PA a longo prazo e não apenas duas horas após a imersão (KWEE et al., 2000). Segundo Katz et al. (1992), um estudo com I I gestantes saudáveis, imersas ao nível da cintura escapular, a $34{ }^{\circ} \mathrm{C}$, por 4 a 5 dias consecutivos, resultou em redução na PA e na FC, com este efeito mantido para cada dia de estudo, contudo os itens foram medidos dentro de 2 horas após a imersão, mas não daí em diante.

Além disso, há relato de possíveis diferenças na PA materna no primeiro trimestre e segundo, por isso investigação adicional é indicada, enfocando gestantes com síndromes hipertensivas (HERMIDA; AYALA; IGLESIAS, 200 I; STREVENS; WIDE-SWENSSON; INGEMARSSON, 200 I).

No quadro I são apresentados os resumos dos estudos supracitados, com as respectivas temperaturas, profundidades de imersão utilizadas e respostas da PA de repouso em imersão.

Quadro I. Comportamento da pressão arterial (PA) durante a imersão em gestantes

\begin{tabular}{|c|c|c|c|c|c|}
\hline Autor & Participantes & $\begin{array}{l}\text { Tempe- } \\
\text { ratura } \\
\text { da água }\end{array}$ & $\begin{array}{l}\text { Tempo de } \\
\text { imersão }\end{array}$ & $\begin{array}{l}\text { Profundidade } \\
\text { de imersão }\end{array}$ & Resultados \\
\hline $\begin{array}{c}\text { Kokot, } \\
\text { Ulman, } \\
\text { Cekanski } \\
\text { (1983) }\end{array}$ & $\begin{array}{l}39 \text { gestantes } \\
\text { saudáveis e } \\
45 \text { com pré- } \\
\text { eclâmpsia } \\
\text { tardia leve ou } \\
\text { moderada }\end{array}$ & $\begin{array}{l}35- \\
36^{\circ} \mathrm{C}\end{array}$ & $\begin{array}{c}60 \\
\text { minutos }\end{array}$ & Pescoço & $\begin{array}{l}\text { Queda rápida e acen- } \\
\text { tuada na PAS e PAD, } \\
\text { significativamente maior } \\
\text { em mulheres com pré- } \\
\text { eclâmpsia tardia. }\end{array}$ \\
\hline $\begin{array}{l}\text { Doniec- } \\
\text { Ulman. } \\
(1987)\end{array}$ & $\begin{array}{l}\text { |2 gestantes } \\
\text { saudáveis, } \\
\text { |4 com pré- } \\
\text { eclâmpsia } \\
\text { leve ou } \\
\text { moderada e } \\
\text { I2 mulheres } \\
\text { não-grávidas }\end{array}$ & $\begin{array}{l}35- \\
36^{\circ} \mathrm{C}\end{array}$ & $\begin{array}{l}120 \\
\text { minutos }\end{array}$ & Pescoço & $\begin{array}{l}\text { A PAM foi normalizada } \\
\text { nas gestantes saudáveis, } \\
\text { diminuiu nas mulheres } \\
\text { não-grávidas, enquanto } \\
\text { nas gestantes com pré- } \\
\text { eclâmpsia mostrou ten- } \\
\text { dência a valores basais. }\end{array}$ \\
\hline $\begin{array}{l}\text { Katz. } \\
(1990)\end{array}$ & $\begin{array}{l}\text { I I gestantes } \\
\text { saudáveis }\end{array}$ & $\begin{array}{l}32+- \\
0,5^{\circ} \mathrm{C}\end{array}$ & $\begin{array}{c}50 \\
\text { minutos }\end{array}$ & $\begin{array}{l}\text { Cintura pél- } \\
\text { vica e cintura } \\
\text { escapular }\end{array}$ & $\begin{array}{l}\text { Maior declínio da PAM } \\
\text { na imersão ao nível da } \\
\text { cintura escapular. }\end{array}$ \\
\hline
\end{tabular}




\begin{tabular}{|c|c|c|c|c|c|}
\hline Autor & Participantes & $\begin{array}{l}\text { Tempe- } \\
\text { ratura } \\
\text { da água }\end{array}$ & $\begin{array}{l}\text { Tempo de } \\
\text { imersão }\end{array}$ & $\begin{array}{l}\text { Profundidade } \\
\text { de imersão }\end{array}$ & Resultados \\
\hline $\begin{array}{l}\text { Katz. } \\
(1992)\end{array}$ & $\begin{array}{l}\text { | I gestantes } \\
\text { saudáveis }\end{array}$ & $\begin{array}{l}\text { Termo- } \\
\text { neutra } \\
(34- \\
\left.35^{\circ} \mathrm{C}\right)\end{array}$ & $\begin{array}{c}50 \\
\text { minutos }\end{array}$ & $\begin{array}{l}\text { Cintura } \\
\text { escapular }\end{array}$ & $\begin{array}{l}\text { Redução na PA e na FC } \\
\text { com este efeito man- } \\
\text { tido para cada dia do } \\
\text { estudo. }\end{array}$ \\
\hline $\begin{array}{l}\text { Kwee. } \\
(2000)\end{array}$ & $\begin{array}{l}19 \text { gestantes } \\
\text { saudáveis }\end{array}$ & $\begin{array}{l}\text { Termo- } \\
\text { neutra } \\
(34- \\
\left.35^{\circ} \mathrm{C}\right)\end{array}$ & 35 minutos & $\begin{array}{l}\text { I, } 10 \mathrm{~m} \mathrm{a} \\
\mathrm{I}, 70 \mathrm{~m}\end{array}$ & $\begin{array}{l}\text { Redução significativa da } \\
\text { PA durante curto período } \\
\text { de tempo ( } 1,5 \text { horas). }\end{array}$ \\
\hline $\begin{array}{l}\text { Hart- } \\
\text { mann. } \\
(200 I)\end{array}$ & $\begin{array}{l}32 \text { gestantes } \\
\text { e } 2 \text { hiper- } \\
\text { tensas }\end{array}$ & $29^{\circ} \mathrm{C}$ & 45 minutos & Axila & $\begin{array}{l}\text { Os valores da PA foram } \\
\text { menores após o exercí- } \\
\text { cio em água do que na } \\
\text { imersão em repouso. }\end{array}$ \\
\hline $\begin{array}{l}\text { Aires. } \\
(2001)\end{array}$ & $\begin{array}{l}52 \text { gestantes } \\
\text { saudáveis }\end{array}$ & $\begin{array}{l}\text { Termo- } \\
\text { neutra } \\
(34- \\
\left.35^{\circ} \mathrm{C}\right)\end{array}$ & $\begin{array}{c}30,45 \text { e } \\
60 \text { minutos }\end{array}$ & $\begin{array}{l}\text { Cintura } \\
\text { escapular }\end{array}$ & $\begin{array}{l}\text { Maior redução significa- } \\
\text { tiva da PAM no grupo } \\
\text { imerso por } 60 \text { minutos }\end{array}$ \\
\hline $\begin{array}{l}\text { Finkels- } \\
\text { tein. } \\
(2004)\end{array}$ & $\begin{array}{l}\text { | | gestantes } \\
\text { saudáveis }\end{array}$ & $31,5^{\circ} \mathrm{C}$ & 7 minutos & $\begin{array}{l}\text { Diferentes } \\
\text { níveis de } \\
\text { imersão }\end{array}$ & $\begin{array}{l}\text { Redução significativa de } \\
\text { I8,7\% para PAS e de } \\
\text { I7,2\% para PAD quando } \\
\text { imersas na profundidade } \\
\text { do processo xifóide. }\end{array}$ \\
\hline $\begin{array}{l}\text { Ward. } \\
(2005)\end{array}$ & $\begin{array}{c}33 \text { gestantes } \\
\text { saudáveis }\end{array}$ & $32^{\circ} \mathrm{C}$ & 60 minutos & $\begin{array}{c}0,9 \mathrm{~m} \text { a } \\
1,4 \mathrm{~m}\end{array}$ & $\begin{array}{l}\text { A PAS, PAD e PAM dimi- } \\
\text { nuíram significativamente } \\
(\mathrm{p}<0,00 \text { I }) \text { de } 2-5 \text { minu- } \\
\text { tos após entrada na água, } \\
\text { no entanto não houve } \\
\text { mudança significativa na } \\
\text { PAM na pré-imersão e } \\
\text { na pós-imersão. }\end{array}$ \\
\hline $\begin{array}{l}\text { Hart- } \\
\text { mann e } \\
\text { Huch. } \\
(2005)\end{array}$ & $\begin{array}{l}9 \text { gestantes } \\
\text { saudáveis }\end{array}$ & $30,9^{\circ} \mathrm{C}$ & 45 minutos & Axila & $\begin{array}{l}\text { A PAS foi significativamen- } \\
\text { te aumentada I mim após } \\
\text { o exercício, enquanto a } \\
\text { PAD permaneceu pra- } \\
\text { ticamente inalterada no } \\
I^{\circ} \text { e no } 5^{\circ} \text { minuto após } \\
\text { o exercício }\end{array}$ \\
\hline
\end{tabular}




\begin{tabular}{|c|c|c|c|c|c|}
\hline Autor & Participantes & $\begin{array}{c}\text { Tempe- } \\
\text { ratura } \\
\text { da água }\end{array}$ & $\begin{array}{l}\text { Tempo de } \\
\text { imersão }\end{array}$ & $\begin{array}{l}\text { Profundidade } \\
\text { de imersão }\end{array}$ & Resultados \\
\hline $\begin{array}{c}\text { Elvan- } \\
\text { Taspinar. } \\
\text { (2006) }\end{array}$ & $\begin{array}{l}7 \text { gestantes } \\
\text { com pré- } \\
\text { eclâmpsia, } \\
7 \text { gestantes } \\
\text { saudáveis e } \\
7 \text { mulheres } \\
\text { não-grávidas }\end{array}$ & $\begin{array}{c}\text { Termo- } \\
\text { neutra } \\
(34- \\
\left.35^{\circ} \mathrm{C}\right)\end{array}$ & $\begin{array}{l}180 \\
\text { minutos }\end{array}$ & $\begin{array}{c}\text { I,20 m até o } \\
\text { nível da fossa } \\
\text { esternoclavi- } \\
\text { cular }\end{array}$ & $\begin{array}{l}\text { A PAS manteve-se inal- } \\
\text { terada nos } 3 \text { grupos, } \\
\text { houve redução da PAD } \\
\text { e da FC. A imersão não } \\
\text { teve efeito na vasodi- } \\
\text { latação do endotélio- } \\
\text { dependente no grupo } \\
\text { de pré-eclâmpsia }\end{array}$ \\
\hline
\end{tabular}

PAS: pressão arterial sistólica; PAD: pressão arterial diastólica; PAM: pressão arterial média; FC: freqüência cardíaca.

\section{CONSIDERAÇÕES FINAIS}

Por anos, acreditou-se que a gestante deveria passar os nove meses em repouso. A maioria dos profissionais da área de saúde temia que a prática de atividade física acarretasse prejuízos para o estabelecimento do ciclo gravídicopuerperal e aconselhavam, por conta disso, o repouso absoluto para todas as gestantes. Felizmente, as pesquisas na área vêm demonstrando os benefícios das atividades realizadas no meio aquático em relação ao bem-estar geral da mulher durante a fase gestacional.

Em sete artigos selecionados nesta pesquisa, as gestantes apresentaram uma diminuição na PA de repouso com a imersão em meio aquático, sendo esta redução proporcional ao nível de imersão, ratificando a influência da mesma sobre a PA e levantando-se a necessidade de ensaios clínicos com mais poder, adequados desenhos metodológicos e aferição de desfechos relevantes, para que a imersão possa se configurar com uma terapêutica bem fundamentada para controle da pressão arterial nas síndromes hipertensivas gestacionais.

A efetividade da imersão na água em gestantes com síndromes hipertensivas ainda é pouco conhecida, devido aos poucos estudos de qualidade disponíveis. Em dez dos estudos selecionados, o experimento foi realizado em única sessão e não a longo prazo, além de que apenas um dos estudos foi randomizado controlado. Por isso, pensamos que futuras investigações devem ser realizadas na prática terapêutica no meio aquático para esclarecer melhor o papel da imersão no comportamento da PA em gestações saudáveis e de risco. 


\section{Repercussion of immersion on blood pressure in pregnant women}

ABSTRACT: We reviewed the changes of $B P$ in pregnant women during immersion to understand the factors that influence this behavior. It is observed that resting BP is reduced by immersion in water, this reduction is proportional to the level of immersion. Factors such as water temperature, body position, depth and immersion time influence the body's physiological responses to this condition. However, the studies have different parameters for these variables, which hinders the establishment of an adequate indication of this modality as a therapeutic resource.

KEYWORDS: Pregnancy; hypertension pregnancy-induced; physical therapy.

\section{Repercusiones de la inmersión en la presión arterial de gestantes}

RESUMEN: Revisamos en la literatura sobre los cambios de la presión arterial en mujeres embarazadas durante la inmersión para comprender los factores que influyen en este comportamiento. Se observa que en reposo se reduce la presión arterial por inmersión en agua, esta reducción es proporcional al nivel de inmersión. Factores como temperatura del agua, posición corporal, profundidad y tiempo de inmersión tienen influencia en las respuestas fisiológicas del organismo a esa condición. Sin embargo, los estudios tienen parámetros diferentes para estas variables, lo que dificulta el establecimiento de una indicación adecuada de esta modalidad como un recurso terapéutico.

PALABRAS CLAVE: Embarazo; hipertensión inducida en el embarazo; terapia física.

\section{REFERÊNCIAS}

American College of Obstetricians and Gynecologists (ACOG). Exercise during pregnancy and the postpartum period. ACOG Technical Bulletin 189. Washington, D.C.: ACOG, 1994.

AYRES, C. E. Modificações no índice de líquido amniótico estimado pela ultra-sonografia em gestantes submetidas à imersão subtotal em água. Revista Brasileira de Ginecologia e Obstetrícia, v. 23, n. 2, p. 101-105, 2001.

ALBERTON, C. L.; KRUEL, L. F. M. Influência da imersão nas respostas cardiorrespiratórias em repouso. Revista Brasileira de Medicina do Esporte, Porto Alegre, v. I 5, n. 3, p. 228-232, mai./jun. 2009.

ASAI, M.. Effect of exercise in water on maternal blood circulation. Nippon Sanka Fujinka Gakkai Zasshi, Japan, v. 46, n. 2, p. 109-114, feb. 1994.

CAROMANO, F. A.; NOWOTNY, J. P. Princípios físicos que fundamentam a hidroterapia. Fisioterapia Brasil, São Paulo, v. 3, n. 6, p. I-9, nov./dez. 2002.

CAVALLI, R. C. et al. Predição de pré-eclampsia (editorial). Revista Brasileira de Ginecologia e Obstetrícia, Ribeirão Preto, v. 3 I, n. I, p. I-4, jan. 2009.

COELHO, B. T.; POLITO, M. D. Efeito agudo de uma sessão de hidroginástica sobre a resposta da pressão arterial em gestantes não hipertensas. Revista da SOCERJ, Londrina, v. 22, n. 2, p. 75-79, mar./abril 2009. 
DONIEC-ULMAN, I. et al. Water immersion-induced endocrine alterations in women with EPH gestosis. Clinical Nephrology, Poland, v. 28, n. 8, p. 5I-55, aug. 1987.

ELVAN-TASPINAR, A. et al. Water immersion in preeclampsia. American Journal of Obstetrics \& Gynecology, Utrecht, v. 195, n. 6, p. 1590-1595, dec. 2006.

ELVAN-TASPINAR, A. et al. Water immersion shows little benefit in preeclampsia. Contemporary OB/GYN, San Diego, jan. 2007.

FINKELSTEIN, I.et al. Comportamento da frequência cardíaca e da pressão arterial, ao longo da gestação, com treinamento no meio líquido. Revista Brasileira de Medicina do Esporte, Porto Alegre, v. 12, n. 5, p. 376-380, set/out. 2006.

FINKELSTEIN, I. et al. Comportamento da Frequência Cardíaca, Pressão Arterial e Peso Hidrostático de Gestantes em Diferentes Profundidades de Imersão. Revista Brasileira de Ginecologia e Obstetrícia, Porto Alegre, v. 26, n. 9, p. 685-690, out. 2004.

GILBERT, J. S. et al. Pathophysiology of hypertension during preeclampsia: linking placental ischemia with endothelial dysfunction. American Journal of Physiology Heart and Circulation Physiology, Mississippi, v. 294, p. 54I-550, feb. 2008.

GRAFA, C. S. The diuretic effect of head-out water immersion and exercise in pregnancy. 1986. 66 f. Dissertação (Mestrado em Ciência) - Faculty of Texas, Tech University, Texas, 1986.

HARTMANN, S.; HUCH, R. Response of pregnancy leg edema to a single immersion exercise session. Acta Obstetricia et Gynecologica Scandinavica, Zurich, v. 84, p. I I 50- I I 53, jan. 2005.

HARTMANN, S. et al. Aqua-fit during pregnancy: maternal and fetal hemodynamic responses during rest, immersion and exercise. Geburtsh Frauenheilk, Zurich, v. 61 , n. 12, p. 977-982, nov. 2001 .

HERMIDA, R. C.; AYALA, D. E.; IGLESIAS, M. Predictable blood pressure variability in healthy and complicated pregnancies. Hypertension, Dallas, v. 38, p. 736-74I, sep. 2001.

IVANOV, S. G.; MARKOVA, L. I. Use of a dry immersion method in the treatment of hypertensive crisis. Kosmicheskaia biologiia I aviakosmicheskaia meditsina, Moskva, v. 24, n. I, p. 40-42, jan/feb. 1990.

KATZ, V. L. Exercise in water during pregnancy. Clinical Obstetrics and Gynecology, Oregon, v. 46, n. 2, p. 432-44I, June 2003.

KATZ, V. L.; McMURRAY, R.; CEFALO, R. C. Aquatic exercise during pregnancy. In: ARTAL, R. M.; WISWELL, R. A.; DRINKWATER, B. L. Exercise in Pregnancy. N. 2. Baltimore: Williams and Wilkins, 1991, p. 27I-276.

KATZ, V. L. et al. Effect of daily immersion on the edema of pregnancy. American Journal of Perinatology, New York, v. 9, n. 4, p. 225-227, jul. 1992.

KATZ, V. L. et al. A comparison of bed rest and immersion for treating the edema of pregnancy. Obstetrics \& Gynecology, North Carolina, v. 75, n. 2, p. |47-I5I, feb. 1990.

KOKOT, F.; ULMAN, J.; CEKANSKI, A. Influence of head-out water immersion on plasma renin activity, aldosterone, vasopressin and blood pressure in late pregnancy toxaemia. Proceedings of the European Dialysis and Transplant Association, v. 20, p. 557-56I, 1983. 
KWEE, A. et al. The effect of immersion on haemodynamic and fetal measures in uncomplicated pregnancies of nulliparous women. British Journal of Obstetrics and Gynaecology, v. 107, p. 663-668, may 2000.

MINISTÉRIO DA SAÚDE. Saúde da família e a atenção pré-natal e puerperal. Informe da Atenção Básica. ano VII, jul./ago. 2006.

PASCOAL, I. F. Hipertensão e gravidez. Revista Brasileira de Hipertensão, v. 9, n. 3, p. 25626I, jul./set. 2002.

PERAÇOLI, J. C.; PARPINELLI, M. A. Síndromes hipertensivas da gestação: identificação de casos graves. Revista Brasileira de Ginecologia e Obstetrícia, v. 27, n. 10, p. 627-634, 2005.

ROGERS, J.; DAVIS, B. A. How risky are hot tubs and saunas for pregnant women? The American Journal of Maternal/Child Nursing, v. 20, n. 3, p. 137-140, 1995.

SMITH, S. A.; MICHEL, Y. A pilot study on the effects of aquatic exercises on discomforts of pregnancy. Journal of Obstetric, Gynecologic and Neonatal Nursing, v. 35, n. 3, p. 3I5-323, mai./jun. 2006.

SORENSEN, T. K. et al. Recreational Physical Activity During Pregnancy and Risk of Preeclampsia. Hypertension, v. 4I, p. I273-1280, 2003.

SOUZA, A. R.; AMORIM, M. R.; COSTA, A. R. Efeitos da nifedipina no tratamento antihipertensivo da pré-eclâmpsia. Acta Médica Portuguesa, v. 21, p. 35।-358, 2008.

STREVENS, H.; WIDE-SWENSSON, D.; INGEMARSSON, I. Blood pressure during pregnancy in a swedish population impact of parity. Acta Obstetricia et Gynecologica Scandinavica, v. 80, n. 9, p. 824-829, sep. 2001.

WARD, E. J. et al. Immediate blood pressure changes and aquatic physiotherapy. Hypertension in Pregnancy, v. 24, p. 93-102, 2005.

Recebido: 21 jun. 2010 Aprovado: 28 ago. 2010

Endereço para correspondência: Leomara Mendes de Oliveira Rua Adalgisa Luna de Menezes, 822 Bairro Bancários João Pessoa - PB CEP: 58.05 I-840 